\title{
openheart Predictors and clinical implication of high-sensitivity cardiac troponin-I elevation following diagnostic cardiac catheterisations
}

Rikuta Hamaya, Taishi Yonetsu, Tadashi Murai, Yoshihisa Kanaji, Eisuke Usui, Junji Matsuda, Masahiro Hoshino, Makoto Araki, Masahiro Hada, Takayuki Niida, Sadamitsu Ichijo, Yoshinori Kanno, Tsunekazu Kakuta

To cite: Hamaya R, Yonetsu T, Murai T, et al. Predictors and clinical implication of high-sensitivity cardiac troponin-I elevation following diagnostic cardiac catheterisations. Open Heart 2017;4:e000586. doi:10.1136/ openhrt-2016-000586

Received 19 December 2016 Revised 27 December 2016 Accepted 3 January 2017

\section{CrossMark}

Division of Cardiovascular Medicine, Tsuchiura Kyodo General Hospital, Ibaraki, Japan

Correspondence to Tsunekazu Kakuta; kaz@joy. email.ne.jp

\section{ABSTRACT}

Objectives Although diagnostic coronary angiography (CAG) is performed worldwide, procedure-related myocardial necrosis (PMN) following diagnostic catheterbased procedures has not been well investigated. The aim of this study was to determine clinical and procedural factors associated with PMN, using a high-sensitivity cardiac troponin I (hs-cTnl) assay, and to investigate the clinical implications of PMN.

Methods Among 697 patients undergoing elective CAG and pre- and post-procedural hs-cTnl (pre-Tnl, post-Tnl, respectively) measurements, a total of 538 patients (124 female) were evaluated, with $2.2 \%$ lost during follow-up. Minor PMN was defined as post-Tnl above the sex-specific upper reference limit (URL), with a $20 \%$ increase from the pre-Tnl level. Major PMN was defined as post-Tnl above $5 x$ the URL. Clinical and procedural factors predicting PMN and the association between PMN and major adverse cardiac events (MACE) following CAG were examined. Results PMN of any type was detected in 178 patients (33.0\%), while major PMN was observed in 32 patients (5.9\%). Female sex, estimated glomerular filtration rate, procedural time, left ventricular end-diastolic pressure (LVEDP) and fractional flow reserve measurement independently predicted any PMN; whereas, only LVEDP and log-transformed $\mathrm{N}$-terminal pro-brain natriuretic peptide independently predicted major PMN. The incidence of MACE was significantly associated with major PMN. Cox proportional-hazards models revealed that major PMN, pre-Tnl, and the absence of statin use were independently associated with MACE.

Conclusions Diagnostic cardiac catheteriation may highlight cardiomyocyte susceptibility to stress in patients with or without CAD. CAG-related major myocardial injury might be associated with future adverse cardiac events independently of the presence or absence of functional stenosis.

\section{INTRODUCTION}

Diagnostic coronary angiography (CAG) has a low rate of complication and is considered the gold standard for the evaluation of coronary artery disease $(\mathrm{CAD}) .^{1}$ More than 10 million CAG procedures are performed annually

\section{KEY QUESTIONS}

What is already known about this subject?

- Coronary angiography (CAG) is an established method for coronary artery disease (CAD) assessment with quite a low rate of complication. However, procedure-related myocardial necrosis (PMN) following diagnostic catheter-based procedures has not been well investigated.

What does this study add?

- We defined PMN by high-sensitivity cardiac troponin I (hs-cTnl) elevation following diagnostic cardiac catheterization using two distinct criteria: any (hs-cTnl> upper reference limit (URL)) and major (hs-cTnl>5*URL) PMN. Any PMN was associated with patient clinical characteristics and catheter-procedural factors including fractional flow reserve measurement, while major PMN was only related to left ventricular end-diastolic pressure. Major PMN was independently linked to the subsequent risk of future adverse cardiac events.

How might this impact on clinical practice?

- Diagnostic cardiac catheteriation may highlight cardiomyocyte susceptibility to stress in patients with or without CAD. CAG-related major myocardial injury might be associated with future adverse cardiac events, suggesting potential utilities of post-procedural hs-cTnl measurement.

around the world and procedure-induced myocardial infarction (MI) is believed to be extremely rare; however, the phenomenon has not been well investigated..$^{23}$ Recent studies on CAG procedure-related myocardial necrosis (PMN) using high-sensitivity cardiac troponin (hs-cTn) assays, which can detect subtle myocardial necrosis, have shown that various clinical characteristics are associated with a hs-cTn increase above the 99th percentile value (upper reference limit; URL).$^{45}$ In addition, 
an adverse impact of hs-cTn elevation on outcomes has been shown in the settings of acute coronary syndrome (ACS) and percutaneous coronary intervention (PCI)-related myocardial injury. ${ }^{6}$ Of note, the critical thresholds for cardiac troponin elevation in PCI-related myocardial injury have been controversial, ranging from $5 \mathrm{x}$ to $70 \mathrm{x}$ of the URL.

Thresholds for CAG-related hs-cTn elevation have not been well evaluated; in addition, the clinical implications remain elusive. ${ }^{4}$ Moreover, catheter-based diagnostic procedures have been diversified with adjunctive or provisional procedures for CAG (eg, left ventriculography (LVG), right heart catheterization (RHC), fractional flow reserve (FFR), optical coherence tomography (OCT), etc.). Although the clinical implications of these diagnostic procedures have been established, the extent of myocardial injury caused by these procedures has not been clarified. Therefore, the aim of this study was to investigate the frequency, risk factors and clinical implications of PMN, as defined by two different sex-specific cut-off values for a well validated high-sensitivity cardiac troponin-I (hs-cTnI) assay, following CAG and adjunctive diagnostic catheteriations. ${ }^{47}$

\section{METHODS}

\section{Patient population}

Patients undergoing scheduled CAG for suspected stable CAD at the Tsuchiura Kyodo General Hospital between June 2014 and February 2016 were prospectively screened. Exclusion criteria included the presence of unstable symptoms (worsening angina or rest angina within a month), MI episode within 30 days before CAG, decompensated heart failure, scheduled myocardial biopsy, chronic kidney disease (CKD) with dialysis, missing patient or procedural data and unavailable informed consent. In addition, in order to define a minor elevation in hs-cTnI due to catheterization (described below), patients who had an elevated hs-cTnI level above the URL before catheterization were excluded from analysis. At our institution, elective diagnostic CAG and PCI are performed separately in the majority of patients with stable CAD; accordingly, no patient undergoing ad hoc PCI was included in this study. The institutional ethics committee approved this study protocol. All patient data and procedural details were obtained from medical records.

\section{Hs-cTnI measurement and definitions of procedure-related myocardial necrosis}

Cardiac troponin-I was assessed using a commercially available hs-cTnI assay (ARCHITECT i2000 ${ }_{\text {SR }}$ STAT hs-cTnI assay, Abbott Laboratories, North Chicago, Illinois, USA) immediately before catheterization and 18-24 $\mathrm{h}$ after the measurement (pre-TnI and post-TnI, respectively). The 99th percentile cut-off values were defined as $32.7 \mathrm{ng} / \mathrm{L}$ for men and $17.9 \mathrm{ng} / \mathrm{L}$ for women; the limit for a blank was $0.6 \mathrm{ng} / \mathrm{L}$; limit of detection was $1.5 \mathrm{ng} / \mathrm{L}$; and coefficient of variation of $10 \%$ was $6 \mathrm{ng} / \mathrm{L}$, as based on a previous community cohort study of Asian healthy individuals. ${ }^{8}$ Two different sex-specific definitions of PMN were used: minor PMN was defined as a post-TnI level greater than the URL that was at least a $20 \%$ increase from the pre-TnI level according to the diagnosis of acute MI in the current guideline; and major PMN was defined as a post-TnI level $>5 x$ the URL, according to the definition of type $4 \mathrm{a} \mathrm{MI}$ in the same guideline. ${ }^{9}$ Patients were accordingly divided into the no PMN group, the any PMN group (patients with minor or major PMN) and the major PMN group. Ischaemic symptoms and ECG changes were not mandatory for the definitions of PMN in the present study.

\section{Procedures}

Diagnostic CAG was performed via a radial, brachial or femoral artery using a $5 \mathrm{Fr}$ sheath, depending on the operator's discretion. An iodine containing contrast agent, iopamidol, was used throughout the study except for patients with a history of allergy to this contrast agent. All patients received a bolus intravenous injection of heparin (3000 IU) before the procedure, followed by a 2000 IU bolus infusion every hour.

Adjunctive and provisional procedures. LVG was performed following CAG if not contraindicated, and left ventricular end-diastolic pressure (LVEDP) was measured before the contrast agent infusion. Adjunctive catheter-based examinations including aortography, bypass graft angiography, RHC, OCT imaging and FFR measurement were performed depending on the physician's discretion. OCT imaging was indicated for patients with prior PCI in whom the angiographical appearance of the stent deserved a detailed observation, or for those enrolled in prospective studies that required OCT examinations. For OCT imaging, a 5Fr guiding catheter (Heartrail, Terumo, Tokyo, Japan) was introduced into the vessel of interest; thereafter, a 0.014-inch guidewire was advanced to the distal area of the vessel. A frequency-domain OCT system (C8-XR ${ }^{\mathrm{TM}}$ OCT imaging catheter and ILUMIEN OCT Intravascular Imaging System, St. Jude Medical, St. Paul, Minnesota, USA) was used with a pull-back speed of $20 \mathrm{~mm} / \mathrm{s}$ during continuous flushing of the contrast medium through a guiding catheter at a rate of $3.5-4.5 \mathrm{~mL} / \mathrm{s}$ for 3-4 s. FFR measurement was indicated for patients with intermediate stenosis in epicardial coronary arteries (30\% to $80 \%$ diameter stenosis). The institutional protocol for coronary physiological tests are described elsewhere. ${ }^{10}$ Briefly, a sensor-tipped coronary guidewire (Pressure Wire, St. Jude Medical, St. Paul, Minnesota, USA) was introduced into the coronary artery of interest after the pressure was zeroed and equalised to the catheter-tip pressure before crossing the lesion. Thereafter, the wire was positioned approximately $8-10 \mathrm{~cm}$ distal to the ostium of the artery across the index stenosis. Intravenous infusion of adenosine 5 '-triphosphate was administered at $160 \mu \mathrm{g} / \mathrm{kg} / \mathrm{min}$ to induce steady-state maximal hyperaemia. Simultaneous 
measurement of the mean aortic pressure $(\mathrm{Pa})$ and mean distal coronary pressure $(\mathrm{Pd})$ was performed in the hyperaemic state, and FFR was calculated as hyperaemic $\mathrm{Pd} /$ hyperemic Pa.

\section{Angiographic analysis}

Coronary stenosis was visually estimated, and quantitative coronary angiography (QCA) was indicated for lesions with $>30 \%$ diameter stenosis. QCA was performed using dedicated off-line so ftware (QAngio XA 7.3, Medis, Leiden, Netherlands); thereafter, coronary lesions with $>50 \%$ diameter stenosis were considered significant and multivessel disease was defined as significant stenosis in $\geq 2$ coronary arteries. In order to assess the complexity of coronary lesions, SYNTAX and GENSINI scores were determined in patients without prior coronary artery bypass grafting (CABG) $\cdot{ }^{11}$ SYNTAX score was calculated using the online version of SYNTAX score calculator (www.syntaxscore.com), while GENSINI score was manually calculated as the sum of all segment scores, which were the multiply of scores representing percent diameter stenosis and scores of each segment. ${ }^{11}$ The incidences of coronary interventions, including PCI and CABG, that were performed within 6 months after CAG, based on CAG results, were also assessed.

\section{Clinical follow-up}

Clinical follow-up data were collected via a review of the medical records and/or telephone interviews. MI was diagnosed based on the third Universal Definition of Myocardial Infarction. ${ }^{7}$ Remote revascularisation was defined as a revascularisation required $>6$ months after the index catheterization, which excluded those required based on the index diagnostic CAG. Major adverse cardiac events (MACE) were defined as a composite of death from any cause, non-fatal spontaneous MI (except those following catheter procedures), hospital admission due to congestive heart failure (CHF), and remote revascularisation.

\section{Statistical analysis}

Categorical data are expressed as frequencies and percentages, and were compared using the $\chi^{2}$ or Fisher's exact test, as appropriate. Continuous variables with normal distribution are expressed as mean $\pm \mathrm{SD}$ and were compared using the Student's t-test. Variables with a non-normal distribution are expressed as median values (25-75th percentiles) and were compared using MannWhitney $\mathrm{U}$ tests. Multivariate logistic regression analysis was performed to determine the predictors of PMN and included factors showing a $p$ value $<0.05$ in univariate analyses, as well as log-transformed N-terminal pro-brain natriuretic peptide (NT-proBNP). The incidence of MACE in patients with/without PMN was assessed using a Kaplan-Meier curve and log-rank test. We constructed Cox proportional-hazards models to estimate the adjusted association between major PMN and the risk of MACE. All variables associated with adverse events obtaining a $p$ value $<0.05$ in univariate analyses, as well as age, sex and log-transformed NT-proBNP, were then tested in a multivariate Cox proportional-hazards regression analysis. All statistical analyses were performed using SPSS V.19.0 (SPSS, Chicago, Illinois, USA). A two-sided $\mathrm{p}$ value $<0.05$ was considered statistically significant.

\section{RESULTS}

\section{Baseline clinical features}

Of 697 prospectively screened patients undergoing scheduled CAG and hs-cTnI evaluation, 14 patients who adjunctively underwent myocardial biopsy, 30 patients with evolving or recent MI, 10 patients with unstable symptoms, 29 patients on maintenance dialysis, 59 patients with pre-TnI above the URL and 17 patients with insufficient clinical data were excluded. Thus, a total of 538 patients, consisting of $414(77.0 \%)$ men and 124 (23.0\%) women, was included in the analysis. Median pre-TnI and post-TnI values were $4 \mathrm{ng} / \mathrm{L}$ (IQR: $3-8 \mathrm{ng} / \mathrm{L}$ ) and $16.5 \mathrm{ng} / \mathrm{L}$ (IQR: 7-39.3 ng/L), respectively. The time interval between the measures was18-24 hours. Absolute change in the hs-cTnI values in the total population was $9 \mathrm{ng} / \mathrm{L}$ (IQR: 2-32 $\mathrm{ng} / \mathrm{L}$ ), and the relative change was $300 \%(150-800 \%)$. PMN of any type was detected in 178 patients $(33.1 \%)$, in whom 32 patients $(5.9 \%)$ had major PMN.

The baseline characteristics for patients with any/ no PMN/major PMN/no major PMN are summarised in table 1. Patients with any PMN were significantly older, and had a significantly higher number of women, non-smokers and prior aspirin users than those without PMN. Patients with any PMN also showed more frequent aortic stenosis and aortic insufficiency, worse renal function, higher pre-TnI levels and less prevalent mitral regurgitation compared with patients without PMN. Women were more prevalent among patients with major PMN than among those without major PMN. Patients with major PMN had been less frequently treated with an anticoagulant or aspirin compared with those without major PMN. More prevalent prior PCI experiences and higher levels of NT-proBNP were observed in patients with major PMN than in those without major PMN. No significant differences between the PMN groups were observed in the echocardiographic findings.

\section{Procedural characteristics and CAG results}

Characteristics of the catheterization procedures and angiographic findings are summarised in table 2. In patients with any PMN, the procedural time was longer, FFR was more frequently performed, and LVEDP was higher than that in patients without PMN. OCT imaging acquisition was not significantly associated with any PMN. Compared with patients without PMN, patients with any PMN had a higher GENSINI score and rate of revascularisation based on the CAG results, especially with PCI. In contrast, there were no statistically significant differences between patients with 
Table 1 Baseline characteristics of the study population

\begin{tabular}{|c|c|c|c|c|c|c|}
\hline & $\begin{array}{l}\text { Any PMN } \\
(n=178)\end{array}$ & $\begin{array}{l}\text { No PMN } \\
(n=360)\end{array}$ & $\mathbf{p}$ & $\begin{array}{l}\text { Major PMN } \\
(n=32)\end{array}$ & $\begin{array}{l}\text { No Major PMN } \\
(n=506)\end{array}$ & $\mathbf{p}$ \\
\hline \multicolumn{7}{|l|}{ Demographics } \\
\hline Age, y & $69.6+9.0$ & $67.3+10.0$ & 0.007 & $70.3+7.8$ & $67.86+9.82$ & ns \\
\hline Female sex & $58(33.1)$ & $65(18.1)$ & $<0.001$ & $12(37.5)$ & $112(22.1)$ & 0.046 \\
\hline Body mass index, $\mathrm{kg} / \mathrm{m}^{2}$ & $24(21.9-26.5)$ & $24.5(22.2-26.6)$ & ns & $23.8(21.8-26)$ & $24.4(22.2-26.6)$ & ns \\
\hline \multicolumn{7}{|c|}{ Cardiovascular risk factors } \\
\hline Hypertension & $126(71.0)$ & $252(70.0)$ & ns & $23(71.9)$ & $355(70.16)$ & ns \\
\hline Diabetes mellitus & $62(34.8)$ & $138(38.3)$ & ns & $11(34.4)$ & $189(37.35)$ & ns \\
\hline Dyslipidemia & $92(51.7)$ & $181(50.23)$ & ns & $15(46.9)$ & $258(50.99)$ & ns \\
\hline Smoking & $110(61.8)$ & $258(71.7)$ & 0.021 & $18(56.3)$ & $350(69.17)$ & ns \\
\hline \multicolumn{7}{|l|}{ Other comorbidities } \\
\hline Aortic stenosis & $8(4.5)$ & $4(1.1)$ & 0.012 & $0(0)$ & $12(2.37)$ & ns \\
\hline Mitral regurgitation & $1(0.6)$ & $13(3.6)$ & 0.037 & $0(0)$ & $14(2.77)$ & ns \\
\hline Aortic insufficiency & $4(2.3)$ & $1(0.33)$ & 0.025 & $1(3.13)$ & $4(0.79)$ & ns \\
\hline Myocardial infarction & 87 (48.9) & $189(52.5)$ & ns & $16(50)$ & $260(51.38)$ & ns \\
\hline Congestive heart failure & $8(4.5)$ & $29(8.1)$ & ns & $0(0)$ & $37(7.31)$ & ns \\
\hline Atrial fibrillation & $16(9.0)$ & $38(10.6)$ & ns & $1(3.1)$ & $53(10.5)$ & ns \\
\hline \multicolumn{7}{|l|}{ Prior operations } \\
\hline $\mathrm{PCl}$ & $120(67.4)$ & $260(72.2)$ & ns & $16(50)$ & 364 (71.94) & 0.008 \\
\hline CABG & $4(2.3)$ & $13(3.6)$ & ns & $0(0)$ & 17 (3.36) & ns \\
\hline \multicolumn{7}{|l|}{ UCG findings } \\
\hline LVEF, \% & $62(53-70)$ & $64(56-68)$ & ns & $61.5(46.5-69.5)$ & $63(56-69)$ & ns \\
\hline LVH & $36(20.2)$ & $50(13.9)$ & ns & $9(28.1)$ & 77 (15.2) & ns \\
\hline \multicolumn{7}{|l|}{ Medications } \\
\hline Beta blocker & 87 (48.9) & $200(55.6)$ & ns & $14(43.8)$ & $273(54.0)$ & ns \\
\hline ARBs/ACE inhibitors & $120(67.4)$ & $238(66.1)$ & ns & $25(78.1)$ & $333(65.8)$ & ns \\
\hline Aspirin & $124(69.7)$ & $281(78.1)$ & 0.034 & $19(59.4)$ & $386(76.3)$ & 0.032 \\
\hline Anticoagulant & $15(8.4)$ & 41 (11.4) & ns & $0(0)$ & $56(11.1)$ & 0.047 \\
\hline Statins & $131(73.6)$ & $268(74.4)$ & ns & $21(65.6)$ & $378(74.7)$ & ns \\
\hline \multicolumn{7}{|l|}{ Bio-humoral variables } \\
\hline $\mathrm{eGFR}, \mathrm{mL} / \mathrm{min} / 1.73 \mathrm{~m}^{2}$ & $\begin{array}{l}64.2 \\
(54.4-75.5)\end{array}$ & $\begin{array}{l}71 \\
(59.2-83.7)\end{array}$ & $<0.001$ & $\begin{array}{l}62.9 \\
(50.7-77.6)\end{array}$ & $\begin{array}{l}68.8 \\
(57.7-80.6)\end{array}$ & ns \\
\hline LDL cholesterol, mg/dL & $91.5(74-108)$ & $88(75-108)$ & ns & $102(72-118.5)$ & $89(74-107)$ & ns \\
\hline HDL cholesterol, mg/dL & $48(39-57)$ & $46(38-56)$ & ns & $49(39.8-60.8)$ & $46(39-56)$ & ns \\
\hline C-reactive protein, $\mathrm{mg} / \mathrm{dL}$ & $0.1(0-0.2)$ & $0.1(0-0.2)$ & ns & $0.1(0-0.1)$ & $0.1(0-0.2)$ & ns \\
\hline NT-proBNP, ng/L & $\begin{array}{l}140 \\
(58-439)\end{array}$ & $\begin{array}{l}126 \\
(53.3-289)\end{array}$ & ns & $\begin{array}{l}358.5 \\
(84.8-681)\end{array}$ & $\begin{array}{l}126 \\
(53.8-307.5)\end{array}$ & 0.003 \\
\hline $\begin{array}{l}\text { Pre-procedural } \\
\text { hs-cTnl, ng/L }\end{array}$ & $6(3-10.3)$ & $4(2-7)$ & $<0.001$ & $5(3-12)$ & $4(3-8)$ & ns \\
\hline $\begin{array}{l}\text { Post-procedural } \\
\text { hs-cTnl, ng/L }\end{array}$ & $\begin{array}{l}60.5 \\
(39.8-100.8)\end{array}$ & $10(6-17)$ & $<0.001$ & $\begin{array}{l}239 \\
(165.8-326.8)\end{array}$ & $15.5(7-32)$ & $<0.001$ \\
\hline $\begin{array}{l}\text { Absolute changes of } \\
\text { hs-cTnl, ng/L }\end{array}$ & $50.5(32.8-96.3)$ & $4.5(1-9)$ & $<0.001$ & $\begin{array}{l}218.5 \\
(153.5-321.3)\end{array}$ & $8(2-26)$ & $<0.001$ \\
\hline
\end{tabular}

Abbreviations: ARBs/ACE inhibitors, angiotensin receptor blockers/angiotensin converting enzyme inhibitors; CABG, coronary artery bypass grafting; HDL, high density lipoprotein; hs-cTnl, high-sensitivity cardiac troponin-I; LDL, low density lipoprotein; LVEF, left ventricular ejection fraction; LVH, left ventricular hypertrophy; NT-proBNP, N-terminal pro brain natriuretic peptide; PCI, percutaneous coronary intervention; PMN, procedure-related myocardial necrosis; UCG, ultrasound cardiography; . 
Interventional cardiology

Table 2 Diagnostic catheterization procedures and CAG results

\begin{tabular}{|c|c|c|c|c|c|c|}
\hline & $\begin{array}{l}\text { Any PMN } \\
(n=178)\end{array}$ & $\begin{array}{l}\text { No PMN } \\
(n=360)\end{array}$ & $\mathbf{P}$ & $\begin{array}{l}\text { Major PMN } \\
(n=32)\end{array}$ & $\begin{array}{l}\text { No major PMN } \\
(n=506)\end{array}$ & $\mathbf{P}$ \\
\hline \multicolumn{7}{|l|}{ Procedural characteristics } \\
\hline Inexperienced operator & $102(57.3)$ & $215(59.7)$ & ns & $17(53.1)$ & $300(59.3)$ & ns \\
\hline \multicolumn{7}{|l|}{ Approach sites } \\
\hline Radial artery & $161(90.5)$ & $326(90.6)$ & ns & $28(87.5)$ & $459(90.7)$ & ns \\
\hline Brachial artery & $17(9.6)$ & $33(9.2)$ & ns & $4(12.5)$ & $46(9.09)$ & ns \\
\hline Femoral artery & $0(0)$ & $1(0.28)$ & ns & $0(0)$ & $1(0.2)$ & ns \\
\hline Right heart catheterization & $18(10.1)$ & $23(6.4)$ & ns & $3(9.38)$ & $38(7.51)$ & ns \\
\hline Fractional flow reserve & $82(46.1)$ & $88(24.4)$ & $<0.001$ & $11(34.4)$ & $159(31.4)$ & ns \\
\hline Optical coherence tomography & $44(24.7)$ & $92(25.6)$ & ns & $5(15.6)$ & $131(25.9)$ & ns \\
\hline Bypass graft arteriography & $4(2.3)$ & $13(3.6)$ & ns & $0(0)$ & $17(3.36)$ & ns \\
\hline Aortography & $7(3.9)$ & $5(1.4)$ & ns & $2(6.25)$ & $10(1.98)$ & ns \\
\hline Left ventriculography & $166(93.3)$ & 331 (91.9) & ns & $29(90.6)$ & $468(92.5)$ & ns \\
\hline Procedural time, $\min$ & $\begin{array}{l}46.1 \\
(35.2-58.1)\end{array}$ & $\begin{array}{l}36 \\
(25.5-47.8)\end{array}$ & $<0.001$ & $\begin{array}{l}41.1 \\
(31.5-58)\end{array}$ & $\begin{array}{l}39.1 \\
(27.3-51.6)\end{array}$ & ns \\
\hline \multicolumn{7}{|l|}{ CAG results } \\
\hline End-diastolic pressure, $\mathrm{mm} \mathrm{Hg}$ & $16(13-20)$ & $14(11-18)$ & $<0.001$ & $17(13-22)$ & $15(11-19)$ & 0.016 \\
\hline SYNTAX score & $2(0-7)$ & $4(0-10)$ & ns & $2.5(0-14.9)$ & $3(0-9)$ & ns \\
\hline GENSINI score & $\begin{array}{l}22 \\
(11-36)\end{array}$ & $\begin{array}{l}28 \\
(14-44)\end{array}$ & 0.013 & $\begin{array}{l}23 \\
(11-53)\end{array}$ & $\begin{array}{l}26 \\
(14-41)\end{array}$ & ns \\
\hline Multivessel disease & $31(17.9)$ & $85(24.5)$ & ns & $9(28.1)$ & $107(21.9)$ & ns \\
\hline Postintervention & $37(20.8)$ & $112(31.1)$ & 0.012 & $10(31.3)$ & $139(27.5)$ & ns \\
\hline Post-PCl & 34 (19.1) & $104(28.9)$ & 0.015 & $9(28.1)$ & $129(25.5)$ & ns \\
\hline Post-CABG & $3(1.7)$ & $8(2.2)$ & ns & $1(3.1)$ & $10(2.0)$ & ns \\
\hline
\end{tabular}

CABG, coronary artery bypass grafting; CAG, coronary angiography; $\mathrm{PCl}$, percutaneous coronary intervention; PMN, procedure-related myocardial necrosis.

Post-intervention/PCI/CABG refers to these interventions performed based on the CAG results within 6 months after CAG procedures.

Table 3 Predictors for Any PMN

\begin{tabular}{|c|c|c|c|c|c|c|}
\hline & \multicolumn{3}{|c|}{ Univariate models } & \multicolumn{3}{|c|}{ Multivariate model } \\
\hline & Odds ratio & $95 \% \mathrm{Cl}$ & $\mathbf{p}$ & Odds ratio & $95 \% \mathrm{Cl}$ & $\mathbf{p}$ \\
\hline Age $>70 y$ & 1.52 & 1.06 to 2.18 & 0.024 & & & \\
\hline Smoking & 0.64 & 0.44 to 0.94 & 0.021 & & & \\
\hline Aspirin use & 0.65 & 0.43 to 0.97 & 0.034 & & & \\
\hline $\mathrm{eGFR}<60 \mathrm{~mL} / \mathrm{min} / 1.73 \mathrm{~m}^{2}$ & 1.85 & 1.27 to 2.71 & 0.002 & & & \\
\hline FFR & 2.64 & 1.81 to 3.86 & $<0.001$ & 2.61 & 1.59 to 4.31 & $<0.001$ \\
\hline Procedural time & 1.03 & 1.02 to 1.04 & $<0.001$ & 1.02 & 1.00 to 1.03 & 0.014 \\
\hline Log-transformed NT-proBNP & 1.18 & 1.03 to 1.35 & 0.017 & & & \\
\hline
\end{tabular}

The multivariate models comprised 497 patients who underwent left ventriculography.

Abbreviations: EDP, end-diastolic pressure; FFR, fractional flow reserve; NT-proBNP, N-terminal pro brain natriuretic peptide; PMN, procedure-related myocardial necrosis. 
Table 4 Predictors for Major PMN

\begin{tabular}{lllllll}
\hline & Univariate models & \multicolumn{3}{c}{ Multivariate model } \\
\hline & Odds ratio & $\mathbf{9 5 \%} \mathbf{C l}$ & $\mathbf{p}$ & Odds ratio & $\mathbf{9 5 \%} \mathbf{~ C l}$ & $\mathbf{p}$ \\
\hline Female sex & 2.111 & 1.00 to 4.45 & 0.05 & & & \\
Prior PCl & 0.39 & 0.19 to 0.80 & 0.01 & & & \\
Aspirin use & 0.454 & 0.22 to 0.95 & 0.035 & & & \\
eGFR $<60 \mathrm{~mL} / \mathrm{min} / 1.73 \mathrm{~m}^{2}$ & 2.074 & 1.01 to 4.26 & 0.047 & & & \\
Log-transformed NT-proBNP & 1.48 & 1.14 to 1.91 & 0.003 & 1.43 & 1.05 to 1.97 & 0.023 \\
EDP & 1.093 & 1.03 to 1.16 & 0.004 & 1.07 & 1.01 to 1.14 & 0.033 \\
\hline
\end{tabular}

The multivariate models comprised 497 patients who underwent left ventriculography.

Abbreviations: EDP, end-diastolic pressure; NT-proBNP, N-terminal pro brain natriuretic peptide; PMN, procedure-related myocardial necrosis.

and without major PMN in the procedural characteristics or CAG results except for LVEDP.

\section{Predictors of PMN}

Independent predictors of PMN are summarised in tables 3 and 4 . According to the multivariate regression analysis, female sex (OR, 2.04; 95\% CI 1.19-3.54; $\mathrm{p}=0.01$ ), procedural time (OR, 1.02; 95\%CI 1.00-1.03; $\mathrm{p}=0.014$ per min), FFR (OR, 2.61; 95\%CI 1.59-4.31; $<<0.001)$ and LVEDP (OR, 1.08; 95\%CI 1.04-1.12; $\mathrm{p}<0.001$ per mmHg) were independent predictors for any PMN. In contrast, the independent predictors for major PMN were LVEDP (OR, 1.09; 95\% CI 1.03-1.16; $\mathrm{p}=0.033$ per $\mathrm{mmHg}$ ) and log-transformed NT-proBNP (OR, 1.43; 95\% CI 1.05$1.97 ; \mathrm{p}=0.023)$.

\section{Clinical outcomes}

The median follow-up period was 322 days (range: 160-450 days), during which 2 patients died, 6 patients needed hospitalisation due to worsening $\mathrm{CHF}$ and 12 patients underwent remote revascularisations. In addition, during follow-up, 12 patients were lost (2.2\%). Figure 1 shows the Kaplan-Meier curve of survival free from MACE stratified by the presence of any/major PMN (figure 1A and B, respectively). Cumulative event numbers for the no PMN, any PMN and major PMN groups were 15 (4.2\%), 8 (4.5\%) and $4(12.5 \%)$, respectively. Patients with major PMN showed a significantly higher MACE rate $\left(\chi^{2}=5.3, p=0.022\right)$. Stepwise multivariate Cox proportional-hazards regression analysis showed that major PMN, pre-TnI, and the absence of statin use were the independent predictors for adverse cardiac events during follow-up (table 5). During catheterization procedures, two patients experienced ventricular fibrillation immediately after OCT imaging; however, PMN was not detected in those two cases. No cases of periprocedural death, acute MI, aortic dissection, major bleeding, stroke or contrast-induced nephropathy were observed.
A

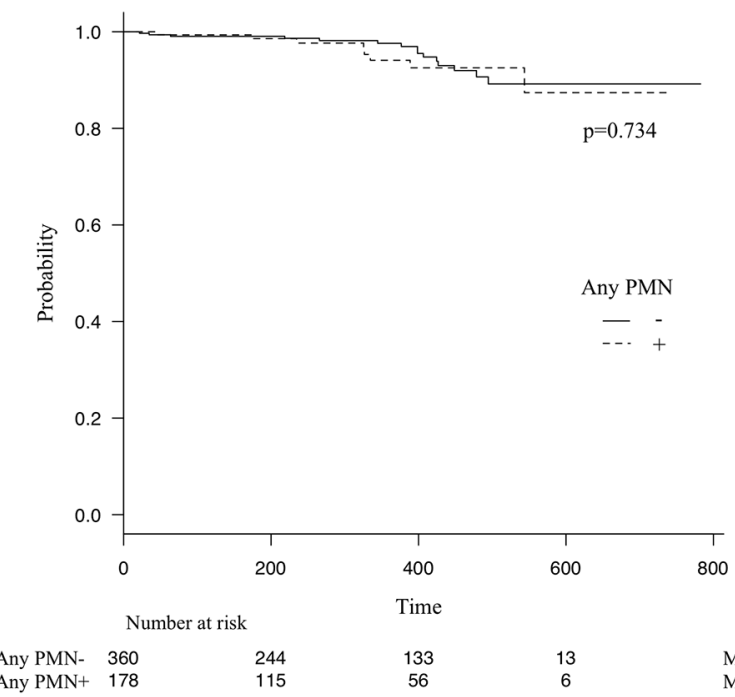

B
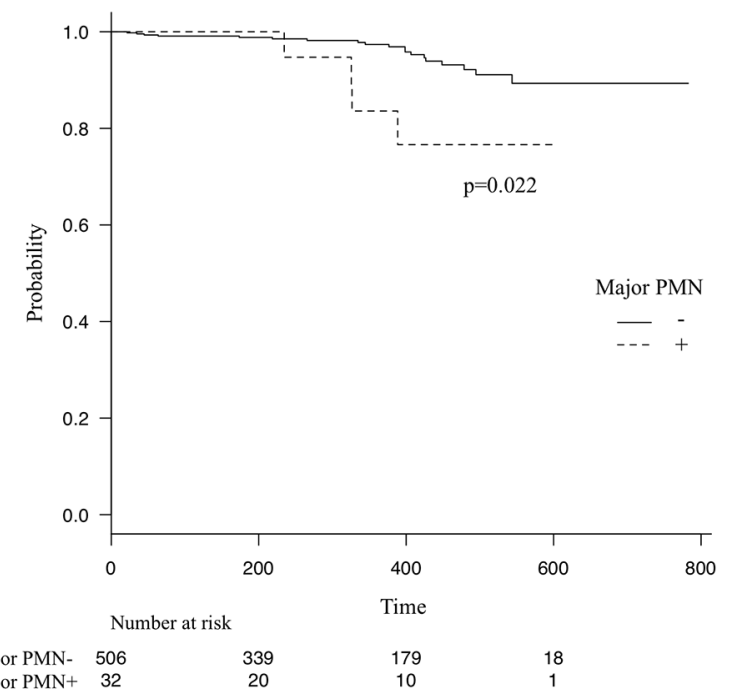

Major PMN- 506
Major PMN+ 32

Figure 1 Kaplan-Meier survival estimates for major cardiovascular events. Curves indicate cumulative survival from major adverse cardiovascular events (MACE) stratified by the presence of any PMN (A) and major PMN (B). Patients with PMN are shown in dotted lines, and patients without PMN are shown in solid lines. MACE was a composite of death from any causes, spontaneous myocardial infraction, hospital admission due to heart failure and remote revascularisation. $P$ values were calculated using log-rank tests. PMN, procedure-related myocardial necrosis. 
Table 5 Hazard ratio for major cardiovascular events

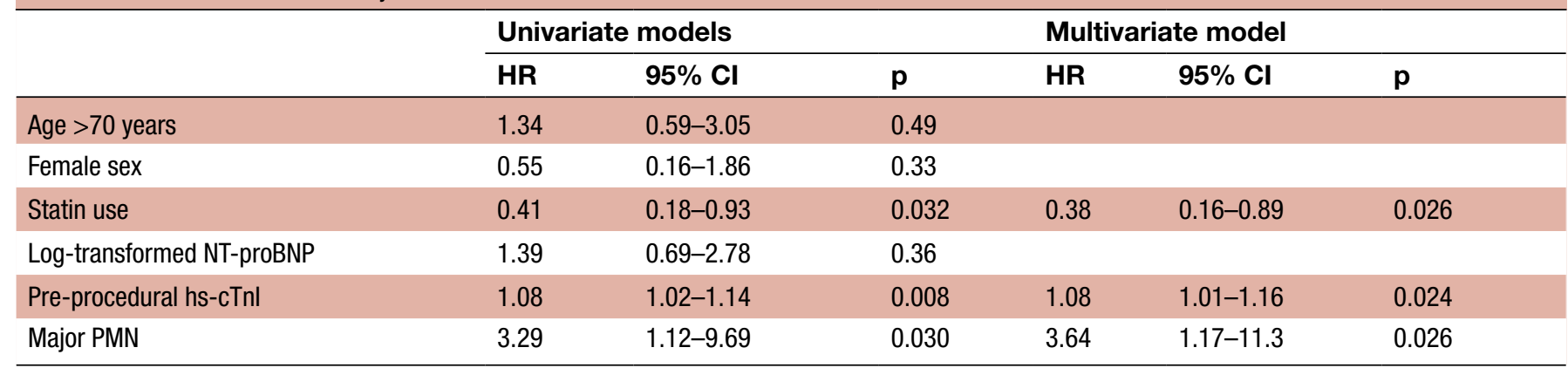

Absence of satin use, preprocedural hs-cTnl, and major PMN were significantly associated with major cardiovascular events; these factors were then put into the multivariate model together with age, sex and log-transformed NT-proBNP.

NT-proBNP, N-terminal pro brain natriuretic peptide; hs-cTnl, high-sensitivity cardiac troponin-l; PMN, procedure-related myocardial necrosis.

\section{DISCUSSION}

The present study is the first to report predictors for, and the incidence and clinical impact of, myocardial necrosis after diagnostic cardiac catheterizations using two different sex-specific hs-cTnI thresholds. We provide the following novel findings: (1) a minor elevation in hs-cTnI above the URL was found in a third of patients, while a major elevation in hs-cTnI consistent with type 4a MI was found in approximately $6 \%$ of patients following diagnostic catheterizations; (2) PMN of any type was associated with patient characteristics and procedural factors including FFR measurement; (3) LVEDP and log-transformed NT-proBNP were the only independent predictors for major PMN; and (4) MACE incidence after diagnostic catheterization was significantly increased in patients with major PMN, but not in those with any PMN.

Cardiac troponins detected by high-sensitive assays are highly specific as well as sensitive markers for myocardial injury, and are recommended in current guidelines for the setting of ACS. ${ }^{79}$ The 99th percentile values based on a normal population, which can only be accurately detected by high-sensitive assays, are important for MI diagnosis, and for predicting adverse long-term outcomes in a number of patient subsets. ${ }^{12}{ }^{13}$ Of note, myocardial injuries should be assessed by high-sensitivity troponin assays, specifically with the well validated URL. As all of the subjects in the present study were Japanese, we selected 9URL from a prior large Asian community cohort study. ${ }^{6}$ In addition, we defined CAG-related troponin elevation by using two definitions: any PMN, which reflected a mild troponin increase beyond the URL; and major PMN, which reflected a major troponin increase above $5 x$ the URL. These different cut-offs were found to have different predictors and clinical implications.

Few studies have investigated CAG-related myocardial injury, especially using hs-cTn; there are only two such prior clinical studies. ${ }^{45}$ In these previous studies, myocardial injury defined as above the URL of high-sensitivity cardiac troponin-T (hs-cTnT) was detected in 13.5\% and $30.3 \%$ of the patients, respectively. In contrast, the present study detected PMN in $33.1 \%$ of the patients. This increased prevalence could be due to the high male prevalence (77\%); frequently added FFR (31.6\%), which was shown to be associated with any PMN; and prolonged procedural time (median, $39.3 \mathrm{~min}$ ) owing to adjunctive procedures. Although 5.9\% of the patients in the present study had an hs-cTnI elevation above the cut-off level for type 4a MI, no cases had chest pain or ECG changes fulfilling the definition of type 4a MI. The frequency of major PMN in the present study is consistent with that in prior reports. $^{35}$

PMN of any type was significantly associated with female sex, eGFR, procedural time, LVEDP and FFR measurement. The association with the former three factors has been implicated in prior studies; however, the most powerful factor appears to be FFR measurement (OR; 2.555). ${ }^{4}{ }^{14}$ Importantly, there was no significant association with other catheter-based examinations including OCT imaging. Both FFR measurement and OCT imaging need wiring in a coronary artery, additional infusion into the artery and additional procedural time. Therefore, the present results suggest that hs-cTnI elevation due to FFR measurement may result from ATP-induced hyperaemia; however, the exact mechanisms of the FFR-related minor hs-cTnI postprocedure increase remain to be determined. In contrast, LVEDP and log-transformed NT-proBNP were the independent predictors for more prominent troponin elevation after CAG. One recent report demonstrated an association between LVEDP and serum hs-cTnT concentration in patients with HF. ${ }^{15}$ Furthermore, CAG-induced troponin elevation could be explained by increased cellular wall permeability or other mechanisms, including ischaemia due to air or thromboembolism, cellular membrane disruption, apoptosis or the formation of membranous blebs. ${ }^{16-21}$ Myocardial stretch may be caused by contrast infusion during CAG, which might lead to the myocyte membrane increasing the permeability of troponins from the cytosol. ${ }^{18}$

Although it has been a matter of debate, prior reports have demonstrated an association between PCI-related MI and long-term adverse outcomes. ${ }^{22}{ }^{23}$ In the present study, major PMN was found to be significantly associated with an increased incidence of long-term MACE. However, no case with major PMN had ischaemic symptoms, ECG 
changes or side branch occlusion after diagnostic catheterization (except for ischaemic symptoms induced by adenosine 5'-triphosphate infusion during FFR measurement). Thus, the troponin elevations were completely subclinical. The poor prognosis clearly did not result directly from myocardial injury caused by diagnostic catheterization; therefore, we hypothesise that a patient status susceptible to CAG-related myocardial injury, for which diagnostic catheterization could cause a significant hs-cTnI elevation, might itself imply the poor long-term prognosis. Elevated baseline troponin is a well known powerful predictor for adverse cardiovascular events. ${ }^{13}$ ${ }^{24}$ The mechanisms of hs-cTn elevation observed in daily activities (ie, increased cellular wall permeability) are possibly similar to those for CAG-related troponin elevation. ${ }^{18} 24$ Additionally, hs-cTnI elevation and baseline hs-cTnI level were found to be independently associated with MACE in the present study, implying the clinical utility of measuring hs-cTnI after catheterization. Prospective cohort studies and further basic studies are needed in order to confirm the prognostic impact and the detailed mechanisms of troponin elevation following diagnostic cardiac catheterization.

\section{LIMITATIONS}

Several limitations of the present study should be considered. First, this was an observational study at a single centre; therefore, selection bias may exist. Second, as serial hs-cTnI change following CAG was not validated, post-TnI could not reflect the maximum value after catheterization. Third, contrast volume data for each patient were not available. There are a number of confounders, such as procedural time, adjunctive procedures and renal sufficiency; however, contrast volume could be significantly associated with CAG-related myocardial necrosis, according to two of three prior studies. ${ }^{45} 14$ Fourth, adjunctive procedures were performed based on the operator's discretion, which could have led to selection bias. Although statistically significant, the implication of the effect of major PMN on adverse clinical outcomes could not be verified without a higher follow-up rate and larger number of patients, or a prospective cohort analysis.

\section{CONCLUSION}

Diagnostic cardiac catheterization may highlight cardiomyocyte susceptibility to stress in patients with or without CAD. The predictors for myocardial injury were different according to the threshold. CAG-related major myocardial injury might be related to future adverse cardiac events.

\section{Competing interests None declared.}

Ethics approval Tsuchiura Kyodo General Hospital Research Ethics Board

Provenance and peer review Not commissioned; externally peer reviewed.

Data sharing statement The authors have detailed clinical information on all included patients. These data would be available to authors with original research proposals after appropriate ethics consultation is complete

Data sharing statement № additional data are available.

Open Access This is an Open Access article distributed in accordance with the Creative Commons Attribution Non Commercial (CC BY-NC 4.0) license, which permits others to distribute, remix, adapt, build upon this work non-commercially, and license their derivative works on different terms, provided the original work is properly cited and the use is non-commercial. See: http://creativecommons.org/ licenses/by-nc/4.0/

(C) Article author(s) (or their employer(s) unless otherwise stated in the text of the article) 2017. All rights reserved. No commercial use is permitted unless otherwise expressly granted.

\section{REFERENCES}

1. Scanlon PJ, Faxon DP, Audet AM, et al. ACC/AHA guidelines for coronary angiography: executive summary and recommendations. A report of the American College of Cardiology/American Heart Association Task Force on Practice Guidelines (Committee on Coronary Angiography) developed in collaboration with the Society for Cardiac Angiography and Interventions. Circulation 1999;99:2345-57.

2. Katritsis D, Korovesis S, Karvouni E, et al. Does diagnostic coronary angiography induce significant coronary microembolization in stable, ischemic patients? A prospective study. J Interv Cardiol 2006;19:346-9.

3. Stefanini GG, Windecker S. Can coronary computed tomography angiography replace invasive angiography? coronary computed tomography angiography cannot replace invasive angiography. Circulation 2015;131:418-25.

4. Kreutzinger P, Wildi K, Twerenbold R, et al. Incidence and predictors of cardiomyocyte injury in elective coronary angiography. Am J Med 2016;129:537.e1-e8.

5. Abu Sharar H, Wohlleben D, Vafaie M, et al. Coronary angiographyrelated myocardial injury as detected by high-sensitivity cardiac troponin T assay. Eurolntervention 2016;12:337-44.

6. Reichlin T, Twerenbold R, Reiter M, et al. Introduction of highsensitivity troponin assays: impact on myocardial infarction incidence and prognosis. Am J Med 2012;125:1205-13.

7. Thygesen K, Alpert JS, Jaffe AS, et al. Third universal definition of myocardial infarction. Eur Heart J 2012;33:2551-67.

8. Aw TC, Phua SK, Tan SP. Measurement of cardiac troponin I in serum with a new high-sensitivity assay in a large multi-ethnic asian cohort and the impact of gender. Clin Chim Acta 2013;422:26-8.

9. Roffi M, Patrono C, Collet JP, et al. ESC guidelines for the management of acute coronary syndromes in patients presenting without persistent ST-segment elevation: Task force for the management of acute coronary syndromes in patients presenting without persistent ST-Segment elevation of the european society of cardiology (ESC). Eur Heart J 2015;2016:267-315.

10. Wakasa N, Kuramochi T, Mihashi N, et al. Impact of pressure signal drift on fractional flow Reserve-Based Decision-Making for patients with intermediate coronary artery stenosis. Circ J 2016;80:1812-9.

11. Sinning C, Lillpopp L, Appelbaum S, et al. Angiographic score assessment improves cardiovascular risk prediction: the clinical value of SYNTAX and gensini application. Clin Res Cardiol 2013;102:495-503.

12. Damman P, Wallentin L, Fox KA, et al. Long-term cardiovascular mortality after procedure-related or spontaneous myocardial infarction in patients with non-ST-segment elevation acute coronary syndrome: a collaborative analysis of individual patient data from the FRISC II, ICTUS, and RITA-3 trials (FIR). Circulation 2012;125:568-76.

13. Everett BM, Brooks MM, Vlachos HE, et al; BARI 2D Study Group. Troponin and cardiac events in stable ischemic heart disease and diabetes. N Engl J Med 2015;373:610-20.

14. Umeda $\mathrm{H}$, Ota $\mathrm{T}$, Iwase $\mathrm{M}$, et al. Subtle myocardial damage associated with diagnostic coronary angiography alone. Eurolntervention 2010;6:388-93.

15. Shionimya $\mathrm{H}$, Koyama $\mathrm{S}$, Tanada $\mathrm{Y}$, et al. Left ventricular enddiastolic pressure and ejection fraction correlate independently with high-sensitivity cardiac troponin-T concentrations in stable heart failure. J Cardiol 2015;65:526-30.

16. Khan M, Schmidt DH, Bajwa T, et al. Coronary air embolism: incidence, severity, and suggested approaches to treatment. Cathet Cardiovasc Diagn 1995;36:313-8.

17. White HD. Pathobiology of troponin elevations: do elevations occur with myocardial ischemia as well as necrosis? J Am Coll Cardiol 2011;57:2406-8. 
18. Hessel MH, Atsma DE, van der Valk EJ, et al. Release of cardiac troponin I from viable cardiomyocytes is mediated by integrin stimulation. Pflugers Arch 2008;455:979-86.

19. Feng J, Schaus BJ, Fallavollita JA, et al. Preload induces troponin I degradation independently of myocardial ischemia. Circulation 2001;103:2035-7.

20. Narula J, Haider N, Virmani R, et al. Apoptosis in Myocytes in endstage heart failure. N Engl J Med 1996;335:1182-9.

21. Schwartz $P$, Piper HM, Spahr R, et al. Ultrastructure of cultured adult myocardial cells during anoxia and reoxygenation. Am J Pathol 1984;115:349-61.
22. Vranckx P, Farooq V, Garg S, et al. Different cardiac biomarkers to detect peri-procedural myocardial infarction in contemporary coronary stent trials: impact on outcome reporting. Heart 2012;98:1424-30.

23. White HD. The prequel: defining prognostically important criteria in the periprocedural PCI troponin saga. Circ Cardiovasc Interv 2012;5:142-5.

24. de Lemos JA, Drazner MH, Omland T, et al. Association of troponin $\mathrm{T}$ detected with a highly sensitive assay and cardiac structure and mortality risk in the general population. JAMA 2010;304:2503-12. 